performed. Patients with IBD diagnosis were identified and searched for VTE diagnosis. Incidence density of VTE was calculated; a case-control study that included IBD patients with and without VTE was performed to analyse VTE related risk factors among Chinese IBD patients.

Results A total of 8459 IBD patients and 46 patients with VTE were identified, yielding an incidence of $37.18 / 10000$ person-years. Risk of VTE increased with advanced age $(\mathrm{RR}=1.05,95 \% \mathrm{CI}=1.02-1.08)$ and history of bowel resection $(\mathrm{RR}=3.52$, 95\%CI=1.45-8.52). Severe disease flare (Crohn's disease activity index $[\mathrm{CDAI}]>450$, mayo score $>10)$, but not disease flares with less severity (CDAI $>150$, mayo $>2$ ), was an independent risk factor for VTE among inpatients (for Crohn's disease: $\mathrm{OR}=11.68,95 \% \mathrm{CI}=2.33-58.47$, ulcerative colitis: $\mathrm{OR}=4.68,95 \% \mathrm{CI}=1.11-19.58)$. In addition, history of glucocorticoids use was a risk factor for patients with Crohn's disease $(\mathrm{OR}=5.01,95 \% \mathrm{CI}=1.22-21.16)$, whereas history of 5aminosalicylic acid use was a protective factor for patients with ulcerative colitis $(\mathrm{OR}=0.16,95 \% \mathrm{CI}=0.04-0.65)$.

Conclusions The incidence of VTE among Chinese IBD patients is not lower than that from the western population. VTE related risk factors included age, severe disease flare, past history of surgery and glucocorticoids use, whereas past use of 5-aminosalicylic acid was protective against VTE for ulcerative colitis. Thromboprophylaxis should be considered for patients with these risk factors.

\section{IDDF2018-ABS-0211 ALTERED COAGULATION IN INFLAMMATORY BOWEL DISEASE: PREDICTORS OF THE DISEASE STATUS}

Juntao Lu*, Lijie Lai, Yuqi Qiao, Dongjuan Song, Jun Shen, Zhihua Ran. Division of Gastroenterology and Hepatology, Key Laboratory of Gastroenterology and Hepatology, Ministry of Health, Inflammatory Bowel Disease Research Center; Renji Hospital, School of Medicine, Shanghai Jiao Tong University; Shanghai Institute of Digestive Disease, Shanghai, China

\subsection{6/gutjnl-2018-IDDFabstracts.156}

Background To evaluate the coagulation status in patients with inflammatory bowel disease (IBD) and explore the possibility of using coagulation parameters to predict the disease status and assist the diagnosis.

Methods We enrolled 987 hospitalised IBD patients and 1027 healthy controls during March 2011 to June 2016. The coagulation parameters between IBD patients and healthy controls were compared. Correlations between coagulation and disease status were evaluated. Computer-based machine learning including artificial neural network (ANN) and support vector machine (SVM), together with receiving operator curve (ROC) was used to estimate the value of coagulation parameters in the diagnosis of IBD.

Results Compared with healthy controls, IBD patients showed higher platelet count and plateletcrit, lower mean platelet volume, platelet distribution width and platelet-large cell ratio, prolonged prothrombin time and activated partial thromboplastin time, and increased fibrinogen and INR $(p<0.001)$. CRP and ESR significantly correlated with hyper-coagulation (nearly all $\mathrm{p}<0.05$ ). In ulcerative colitis (UC) patients, coagulation parameters demonstrated significant correlation with clinical activity and endoscopic activity. Multiple linear regression analysis revealed that in the Crohn's disease (CD) group, patients with colon involvement displayed more obviously altered coagulation status (all $\mathrm{p}<0.05$ ). Structuring lesion and perianal disease were also related with part of the parameters. In UC patients, abnormality of coagulation status was positively paralleled with disease extent. ROC analysis demonstrated that coagulation parameters were of medium predictive value for diagnosing IBD since the area under ROC was 0.748. The overall accuracy of the ANN model was 0.75 with the sensitivity of $73.3 \%$ and the specificity of $76.5 \%$ while in the SVM model, the overall accuracy was 0.744 with the sensitivity of $67.4 \%$ and the specificity of $77.0 \%$.

Conclusions Hyper-coagulation is present in IBD patients. Disease status and coagulation are closely correlated. In CD patients, coagulation parameters are able to indicate the level of inflammatory biomarkers, the location of the lesion, disease behaviour as well as the presence of perianal disease while in UC patients, coagulation parameters are indicative of the the level of inflammatory biomarkers, endoscopic activity and disease extent.

\section{IDDF2018-ABS-0212 PANCREATICOJEJUNOSTOMY WITH INTRAOPERATIVE PANCREATIC STENTING IN WHIPPLE'S PROCEDURE - A SINGLE INSTITUTE EXPERIENCE}

Vijay Kumar*. Additional Professor, Department of Surgical Oncology, King George's Medical University, Lucknow, India

\subsection{6/gutjnl-2018-IDDFabstracts. 157}

Background Pancreaticojejunal anastomotic leak is one of the dreaded complications after whipple's procedure. Many surgical modifications of pancreatico-jejunal anastomosis have been described in the literature, but none are absolutely perfect. We aim to review our recent experience of whipple's procedure with a technique of pancreatico-jejunostomy with intraoperative intrapancreatic stent placement and the postoperative procedure related morbidities and mortality.

Methods In this retrospective study, patients undergoing whipple's procedure for periampullary carcinoma and reconstructed with standard two layered pancreaticojejunostomy with pancreatic stent placement were analyzad from December 2015 to March 2018 and compared with standard technique. Preoperative characteristics of the patients as well as a postoperative pancreatic leak, its morbidity and mortality were compared.

Results During the study period, 33 patients underwent the procedure. Median preoperative bilirubin and serum protein were $4.1 \mathrm{mg} / \mathrm{dl}$ and $7.35 \mathrm{gm} / \mathrm{dl}$. Preoperative Common bile duct stenting was done in 5 patients and 26 patients were jaundiced. Median pancreatic duct diameter was $3.2 \mathrm{~mm}$. The median duration of surgery was 5 hours. There was one patient with postoperative pancreatic leak $(3 \%)$ with was managed conservatively. The median postoperative hospital stay was 15 days. Five patients required ICU care postoperatively. There was no postoperative procedure-related mortality.

Conclusions Pancreaticojejunostomy with intrapancreatic stent placement is a viable option with low rate of postoperative pancreatic leak, its morbidities and mortality in cases of whipple's procedure. 